Article

\title{
Fuzzy Positive Implicative Filters of Hoops Based on Fuzzy Points
}

\author{
Rajab Ali Borzooei ${ }^{1}{ }^{\infty}$, Mona Aaly Kologani ${ }^{2}$, Mahdi Sabet Kish ${ }^{1}$ and Young Bae Jun ${ }^{1,3, *}$ \\ 1 Department of Mathematics, Shahid Beheshti University, Tehran 7561, Iran; borzooei@sbu.ac.ir (R.A.B.); \\ m.sabetkish@sbu.ac.ir (M.S.K.) \\ 2 Hatef Higher Education Institute, Zahedan 8301, Iran; MA.kologani@hatef.ac.ir \\ 3 Department of Mathematics Education, Gyeongsang National University, Jinju 52828, Korea \\ * Correspondence: ybjun@gnu.ac.kr or skywine@gmail.com
}

Received: 14 May 2019; Accepted: 20 June 2019; Published: 24 June 2019

\begin{abstract}
In this paper, we introduce the notions of $(\epsilon, \in)$-fuzzy positive implicative filters and $(\in, \in \vee q)$-fuzzy positive implicative filters in hoops and investigate their properties. We also define some equivalent definitions of them, and then we use the congruence relation on hoop defined in blue [Aaly Kologani, M.; Mohseni Takallo, M.; Kim, H.S. Fuzzy filters of hoops based on fuzzy points. Mathematics. 2019, 7, 430; doi:10.3390/math7050430] by using an $(\in, \in)$-fuzzy filter in hoop. We show that the quotient structure of this relation is a Brouwerian semilattice.
\end{abstract}

Keywords: hoop; sub-hoop; fuzzy points; fuzzy positive implicative filter; Brouwerian semilattice

\section{Introduction}

Hoop is introduced by blueBosbach in [1], and it is naturally ordered commutative residuated integral monoids and he investigated some properties of it in [2]. Then some researchers studied hoops in different was. For example, Blok [3,4], investigated structure of hoops and their applicational reducts. blueBorzooei and Aaly Kologani in [5] defined (implicative, positive implicative, fantastic) filters in a hoop and discussed their relations and properties. Using filter, they considered a congruence relation on a hoop, and induced the quotient structure which is a hoop. They also provided conditions for the quotient structure to be Brouwerian semilattice, Heyting algebra and Wajesberg hoop. After that in [6], they studied these notions in pseudo-hoops. Moreover, in [7,8], researchers investigated n-fold filters, nodal filters and etc, on hoops. Several properties of hoops are displayed in [3-11]. The idea of quasi-coincidence of a fuzzy point with a fuzzy set is mentioned in [12], and it played a vital role to generate some different types of fuzzy subalgebras in of $B C K / B C I$-algebras, called on $(\alpha, \beta)$-fuzzy subalgerbas of $B C K / B C I$-algebras which is introduced by Jun [13]. In particular, $(\in, \in \vee q)$-fuzzy subalgebra is an important and useful generalization of a fuzzy subalgebra in $B C K / B C I$-algebras. Because of that other researchers worked on this topic in different way. For example Redefined fuzzy subalgebra (with thresholds) of BCK/BCI-algebras are studied by Borouman Saied in [14] and Chiranjibe in [15], investigated $(\in, \in \vee q)$-bipolar fuzzy BCK/BCI-algebras, and he extended these notions in [16]. Also, Bakhshi in [17], investigated $(\alpha, \beta)$-fuzzy ideals in pseudo MV-algebras and m-polar $(\alpha, \beta)$-fuzzy ideals in BCK/BCI-algebras is studied by Al-Masarwah in [18]. Moreover, in [19], Aaly introduced the notions of $(\in, \in)$-fuzzy filters and $(\in, \in \vee q)$-fuzzy filters on hoops and disscussed some properties of them. Then they used these notions and defined a congruence relation on hoops and proved that the quotient structure that is made by this relation is a hoop. It is now natural to consider similar style of generalizations of the existing fuzzy subsystems of other algebraic structures. For this reason, we decided to define and investigated these notions on hoop algebras, which we studied [20-23] for sources of inspiration and ideas for this paper. 
The purpose of this paper is define the concepts of $(\in, \in)$-fuzzy positive implicative filters and $(\in, \in \vee q)$-fuzzy positive implicative filters of hoops, by inspiring the concepts of $(\in, \in)((\in, \in$ $\vee q)$ )-fuzzy filters, and some properties of them are investigated. Then we defined some equivalent definitions of them and used the congruence relation on hoop defined in [19] by an $(\epsilon, \in)$-fuzzy filter of hoop, and proved that the quotient structure that is made by this relation is a Brouwerian semilattice.

\section{Preliminaries}

A hoop is an algebra $(H, \odot, \rightarrow, 1)$ in which $(H, \odot, 1)$ is a commutative monoid with the following conditions:

(H1) $x \rightarrow x=1$,

(H2) $x \odot(x \rightarrow y)=y \odot(y \rightarrow x)$,

(H3) $x \rightarrow(y \rightarrow z)=(x \odot y) \rightarrow z$

for all $x, y, z \in H$.

We define a relation " $\leq$ " on a hoop $H$ by

$$
x \leq y \text { if and only if } x \rightarrow y=1
$$

for all $x, y \in H$. It is easy to see that " $\leq$ " is a partial order relation on $H$. A hoop $H$ is said to be bounded if there is an element $0 \in A$ such that $0 \leq x$ for all $x \in A$. Let $x^{0}=1, x^{n}=x^{n-1} \odot x$ for any $n \in \mathbb{N}$. In a bounded hoop $H$, the negation "' " of $x \in H$ is defined by $x^{\prime}=x \rightarrow 0$. A non-empty subset $S$ of a hoop $H$ is called a sub-hoop of $H$ if $x \odot y \in S$ and $x \rightarrow y \in S$ for all $x, y \in S$.

Note that if $S$ is a sub-hoop of a hoop $H$, then $1 \in S$.

Definition 1. [24] Let $(H, \odot, \rightarrow, 1)$ be a bounded hoop. Then the following conditions hold, for all $x, y, z \in H$ :

(i) $(H, \leq)$ is a meet-semilattice with $x \wedge y=x \odot(x \rightarrow y)$,

(ii) $x \odot y \leq z$ if and only if $x \leq y \rightarrow z$,

(iii) $x \odot y \leq x$, $y$ and $x^{n} \leq x$, for any $n \in \mathbb{N}$,

(iv) $x \leq y \rightarrow x$,

(v) $1 \rightarrow x=x$ and $x \rightarrow 1=1$,

(vi) $x \leq(x \rightarrow y) \rightarrow y$,

(vii) $x \rightarrow y \leq(y \rightarrow z) \rightarrow(x \rightarrow z)$,

(viii) $x \leq y$ implies $x \odot z \leq y \odot z, z \rightarrow x \leq z \rightarrow y$, and $y \rightarrow z \leq x \rightarrow z$,

(ix) $((y \rightarrow x) \rightarrow x) \rightarrow x=y \rightarrow x$,

(x) $x^{\prime} \leq x \rightarrow y$ and $x^{\prime \prime \prime}=x^{\prime}$.

Proposition 1. [24] Let $H$ be a hoop and for any $x, y \in H$, we define,

$$
x \vee y=((x \rightarrow y) \rightarrow y) \wedge((y \rightarrow x) \rightarrow x)
$$

If $\vee$ is the join operation on $H$, then hoop $H$ is called a $\vee$-hoop such that $(H, \vee, \wedge)$ is a distributive lattice.

A subset $F$ of a hoop $H$ is called a filter of $H$ if for any $x, y \in F, x \odot y \in F$ and, for any $y \in H$ and $x \in F$, if $x \leq y$, then $y \in F$ (See [24]).

Also, a non-empty subset $F$ of a hoop $H$ is called an implicative filter of $H$ if $1 \in F$, and, for any $x, y, z \in H$, if $x \rightarrow((y \rightarrow z) \rightarrow y) \in F$ and $x \in F$, then $y \in F$. Moreover, a non-empty subset $F$ of a hoop $H$ is called a positive implicative filter of $H$ if $1 \in F$, and for any $x, y, z \in H$, if $x \rightarrow(y \rightarrow z) \in F$ and $x \rightarrow y \in F$, then $x \rightarrow z \in F$ (See [5]). 
Definition 2. [19] Let $(\alpha, \beta)$ be any one of $(\in, \in)$ and $(\in, \in \vee q)$. A fuzzy set $\lambda$ in $H$ is called an $(\alpha, \beta)$-fuzzy filter of $H$ if the following conditions hold.

$$
\begin{aligned}
& (\forall x \in H)(\forall t \in(0,1])\left(x_{t} \alpha \lambda \Rightarrow 1_{t} \beta \lambda\right), \\
& (\forall x, y \in H)(\forall t, k \in(0,1])\left(x_{t} \alpha \lambda,(x \rightarrow y)_{k} \alpha \lambda \Rightarrow y_{\min \{t, k\}} \beta \lambda\right) .
\end{aligned}
$$

A fuzzy set $\lambda$ in a set $X$ of the form

$$
\lambda(y):= \begin{cases}t \in(0,1] & \text { if } y=x \\ 0 & \text { if } y \neq x\end{cases}
$$

is called a fuzzy point with the value $t$ and support $x$, and it is denoted by $x_{t}$.

For a fuzzy set $\lambda$ in a set $X$ and a fuzzy point $x_{t}, \mathrm{Pu}$ and Liu [12] gave meaning to the symbol $x_{t} \alpha \lambda$, where $\alpha \in\{\in, q, \in \vee q, \in \wedge q\}$.

To say that $x_{t} \in \lambda$ (resp. $x_{t} q \lambda$ ) means that $\lambda(x) \geq t$ (resp. $\lambda(x)+t>1$ ), and in this case, $x_{t}$ is said to belong to (resp. be quasi-coincident with) a fuzzy set $\lambda$.

To say that $x_{t} \in \vee q \lambda$ (resp. $x_{t} \in \wedge q \lambda$ ) means that $x_{t} \in \lambda$ or $x_{t} q \lambda$ (resp. $x_{t} \in \lambda$ and $x_{t} q \lambda$ ).

For any fuzzy set $\lambda$ in $H$ and $t \in(0,1]$, we consider the following sets so called $\in$-level set, $q$-set and $\in \vee q$-set, respectively.

$$
\begin{aligned}
& U(\lambda ; t):=\{x \in H \mid \lambda(x) \geq t\}, \\
& \lambda_{q}^{t}:=\left\{x \in H \mid x_{t} q \lambda\right\} \\
& \lambda_{\in \vee q}^{t}:=\left\{x \in H \mid x_{t} \in \vee q \lambda\right\} .
\end{aligned}
$$

Theorem 1. [19] For any $(\in, \in)$-fuzzy filter $\lambda$ of $H, x, y \in H$ and $t, k \in(0,1]$, define

$$
x \equiv_{\lambda} y \text { if and only if }(x \rightarrow y)_{t} \in \lambda \text { and }(y \rightarrow x)_{k} \in \lambda
$$

$\equiv_{\lambda}$ is a congruence relation on $H$. Then $\frac{H}{\equiv_{\lambda}}=\left\{[a]_{\lambda} \mid a \in H\right\}$, in which two operations $\otimes$ and $\rightsquigarrow$ are defined by

$$
[a]_{\lambda} \otimes[b]_{\lambda}=[a \odot b]_{\lambda} \text { and }[a]_{\lambda} \rightsquigarrow[b]_{\lambda}=[a \rightarrow b]_{\lambda}
$$

Then $\left(\frac{H}{\equiv_{\lambda}}, \otimes, \rightsquigarrow,[1]_{\lambda}\right)$ is a hoop such that

$$
[a]_{\lambda} \leq[b]_{\lambda} \text { if and only if }(a \rightarrow b)_{t} \in \lambda, \text { for any } a, b \in H \text { and } t \in(0,1]
$$

Corollary 1. [19] Every $(\in, \in)$-fuzzy filter of $H$ such as $\lambda$ satisfies the following assertion:

$$
(\forall x, y \in H)(\text { if } x \leq y, \text { then } \lambda(x) \leq \lambda(y))
$$

Definition 3. [25] Let $(\alpha, \beta)$ be any one of $(\in, \in)$ and $(\in, \in \vee q)$. A fuzzy set $\lambda$ in $H$ is called an $(\alpha, \beta)$-fuzzy implicative filter of $H$ if

$$
\begin{aligned}
& (\forall x \in H)(\forall t \in(0,1])\left(x_{t} \alpha \lambda \Rightarrow 1_{t} \beta \lambda\right), \\
& (\forall x, y \in H)(\forall t, k \in(0,1])\left(x_{t} \alpha \lambda,(x \rightarrow((y \rightarrow z) \rightarrow y))_{k} \alpha \lambda \Rightarrow y_{\min \{t, k\}} \beta \lambda\right) .
\end{aligned}
$$

Theorem 2. [25] Every $(\in, \in)$-fuzzy implicative filter of $H$ is an $(\in, \in)$-fuzzy filter of $H$.

Proposition 2. [25] If $\lambda$ is an $(\in, \in \vee q)$-fuzzy implicative filter of $H$, then

$$
\min \{\lambda(x \rightarrow(x \rightarrow y)), 0.5\} \leq \lambda(x \rightarrow y)
$$


for any $x, y \in H$.

3. $(\alpha, \beta)$-Fuzzy Positive Implicative Filters for $(\alpha, \beta) \in\{(\in, \in \vee q),(\in, \in)\}$

In this section, we define $(\alpha, \beta)$-fuzzy positive implicative filters for $(\alpha, \beta) \in\{(\in, \in \vee q),(\in, \in)\}$ of hoops and we investigate some properties of them and find some equivalence definitions of them. Also, we investigate the relation between $(\alpha, \beta)$-positive implicative with $(\alpha, \beta)$-implicative one and finally study about the quotient that is made by them.

In what follows, let $H$ denote a bounded hoop unless otherwise specified.

Definition 4. Let $(\alpha, \beta)$ be any one of $(\epsilon, \epsilon)$ and $(\epsilon, \in \vee q)$. A fuzzy set $\lambda$ in $H$ is called an $(\alpha, \beta)$-fuzzy positive implicative filter of $H$ if

$$
\begin{aligned}
& (\forall x \in H)(\forall t \in(0,1])\left(x_{t} \alpha \lambda \Rightarrow 1_{t} \beta \lambda\right), \\
& (\forall x, y \in H)(\forall k, t \in(0,1])\left((x \rightarrow(y \rightarrow z))_{t} \alpha \lambda,(x \rightarrow y)_{k} \alpha \lambda \Rightarrow(x \rightarrow z)_{\min \{t, k\}} \beta \lambda\right) .
\end{aligned}
$$

Example 1. Let $H=\{0, a, b, 1\}$ be a set with the following Cayley tables.

\begin{tabular}{l|llll}
$\rightarrow$ & 0 & $a$ & $b$ & 1 \\
\hline 0 & 1 & 1 & 1 & 1 \\
$a$ & $a$ & 1 & 1 & 1 \\
$b$ & 0 & $a$ & 1 & 1 \\
1 & 0 & $a$ & $b$ & 1
\end{tabular}

\begin{tabular}{l|llll}
$\odot$ & 0 & $a$ & $b$ & 1 \\
\hline 0 & 0 & 0 & 0 & 0 \\
$a$ & 0 & 0 & $a$ & $a$ \\
$b$ & 0 & $a$ & $b$ & $b$ \\
1 & 0 & $a$ & $b$ & 1
\end{tabular}

By routine calculations we chack that $(H, \odot, \rightarrow, 0,1)$ is a bounded hoop. Define $\lambda(0)=0.6, \lambda(a)=$ $0.4, \lambda(b)=0.55$ and $\lambda(1)=0.8$. It is easy to see that $\lambda$ is an $(\in, \in)$-fuzzy positive implicative filter of $H$.

Theorem 3. A fuzzy set $\lambda$ in $H$ is an $(\in, \in)$-fuzzy positive implicative filter of $H$ if and only if it satisfies:

$$
\begin{aligned}
& (\forall x \in H)(\lambda(1) \geq \lambda(x)), \\
& (\forall x, y \in H)(\lambda(x \rightarrow z) \geq \min \{\lambda(x \rightarrow y), \lambda(x \rightarrow(y \rightarrow z))\}) .
\end{aligned}
$$

Proof. Let $\lambda$ be an $(\in, \in)$-fuzzy positive implicative filter of $H, t \in(0,1]$ and $x \in H$ such that $\lambda(x)=t$. Since $\lambda$ is an $(\in, \in)$-fuzzy positive implicative filter of $H$, we have $\lambda(1) \geq t=\lambda(x)$. Hence, for any $x \in H, \lambda(1) \geq \lambda(x)$. Now, let $x, y, z \in H$ and $t, k \in(0,1]$ such that $\lambda(x \rightarrow y) \geq t$ and $\lambda(x \rightarrow(y \rightarrow z)) \geq k$. So $(x \rightarrow y)_{t} \in \lambda$ and $(x \rightarrow(y \rightarrow z))_{k} \in \lambda$. Since $\lambda$ is an $(\in, \in)$-fuzzy positive implicative filter of $H,(x \rightarrow z)_{\min \{t, k\}} \in \lambda$, and so $\lambda(x \rightarrow z) \geq \min \{t, k\}$. Hence,

$$
\min \{\lambda(x \rightarrow(y \rightarrow z)), \lambda(x \rightarrow y)\} \leq \lambda(x \rightarrow z)
$$

Conversely, let $x \in H$ and $t \in(0,1]$ such that $x_{t} \in \lambda$. Then $\lambda(x) \geq t$. Since $t \leq \lambda(x) \leq \lambda(1)$, we have $1_{t} \in \lambda$. Now, assume that $(x \rightarrow y)_{t} \in \lambda$ and $(x \rightarrow(y \rightarrow z))_{k} \in \lambda$ for any $x, y, z \in H$ and $t, k \in(0,1]$. Then by assumption,

$$
\min \{t, k\} \leq \min \{\lambda(x \rightarrow(y \rightarrow z)), \lambda(x \rightarrow y)\} \leq \lambda(x \rightarrow z)
$$

and so $\min \{t, k\} \leq \lambda(x \rightarrow z)$. Hence, $(x \rightarrow z)_{\min \{t, k\}} \in \lambda$. Therefore, $\lambda$ is an $(\in, \in)$-fuzzy positive implicative filter of $H$

Theorem 4. Every $(\in, \in)$-fuzzy positive implicative filter of $H$ is an $(\in, \in)$-fuzzy filter of $H$. 
Proof. Let $\lambda$ be an $(\in, \in)$-fuzzy positive implicative filter of $H$. Then it is clear that if $x_{t} \in \lambda$, then $1_{t} \in \lambda$, for any $x \in H$ and $t \in(0,1]$. Now, let $x, y \in H$ and $t, k \in(0,1]$ such that $x_{t} \in \lambda$ and $(x \rightarrow y)_{k} \in \lambda$. So, $(1 \rightarrow x)_{t} \in \lambda$ and $(1 \rightarrow(x \rightarrow y))_{k} \in \lambda$. Since $\lambda$ is an $(\in, \in)$-fuzzy positive implicative filter of $H,(1 \rightarrow y)_{\min \{t, k\}}=y_{\min \{t, k\}} \in \lambda$. Hence, $\lambda$ is an $(\in, \in)$-fuzzy filter of $H$.

In the following example we show that the converse of Theorem 4, may not be true, in general.

Example 2. Let $H=\{0, a, b, c, d, 1\}$ be a set. Define the binary operations $\odot$ and $\rightarrow$ on $H$ as follows:

\begin{tabular}{l|llllll}
$\rightarrow$ & 0 & $a$ & $b$ & $c$ & $d$ & 1 \\
\hline 0 & 1 & 1 & 1 & 1 & 1 & 1 \\
$a$ & $c$ & 1 & $b$ & $c$ & $b$ & 1 \\
$b$ & $d$ & $a$ & 1 & $b$ & $a$ & 1 \\
$c$ & $a$ & $a$ & 1 & 1 & $a$ & 1 \\
$d$ & $b$ & 1 & 1 & $b$ & 1 & 1 \\
1 & 0 & $a$ & $b$ & $c$ & $d$ & 1
\end{tabular}

\begin{tabular}{l|llllll}
$\odot$ & 0 & $a$ & $b$ & $c$ & $d$ & 1 \\
\hline 0 & 0 & 0 & 0 & 0 & 0 & 0 \\
$a$ & 0 & $a$ & $d$ & 0 & $d$ & $a$ \\
$b$ & 0 & $d$ & $c$ & $c$ & 0 & $b$ \\
$c$ & 0 & 0 & $c$ & $c$ & 0 & $c$ \\
$d$ & 0 & $d$ & 0 & 0 & 0 & $d$ \\
1 & 0 & $a$ & $b$ & $c$ & $d$ & 1
\end{tabular}

Then $(H, \odot, \rightarrow, 0,1)$ is a bounded hoop. Define a fuzzy set $\lambda$ in $H$ as follows:

$$
\lambda: H \rightarrow[0,1], x \mapsto\left\{\begin{array}{l}
0.5 \text { if } x=0, \\
0.7 \text { if } x=a, \\
0.3 \text { if } x=b, \\
0.5 \text { if } x=c \\
0.3 \text { if } x=d, \\
0.8 \text { if } x=1
\end{array}\right.
$$

It is routine to verify that $\lambda$ is an $(\in, \in)$-fuzzy positive implicative filter of $H$ but it is not an $(\in, \in)$-fuzzy filter of $H$ since

$$
0.3=\lambda(b) \nsupseteq \min \{\lambda(0), \lambda(0 \rightarrow b)\}=\min \{0.5,0.8\}
$$

Corollary 2. Every $(\in, \in)$-fuzzy positive implicative filter of $H$ such as $\lambda$ satisfies the following assertion:

$$
(\forall x, y \in H)(\text { if } x \leq y, \text { then } \lambda(x) \leq \lambda(y))
$$

Theorem 5. Given a non-zero $(\in, \in)$-fuzzy positive implicative filter $\lambda$ of $H$, the next set

$$
H_{0}:=\{x \in H \mid \lambda(x) \neq 0\}
$$

is a positive implicative filter of $H$.

Proof. Let $x \in H_{0}$. Since $\lambda(x) \neq 0$, we get $\lambda(x) \geq t$ for some $t \in(0,1]$. Moreover, from $\lambda$ is an $(\in, \in)$-fuzzy positive implicative filter of $H$ and $x_{t} \in \lambda$, we have $1_{t} \in \lambda$. Then $\lambda(1) \geq \lambda(x)=t \neq 0$, and so $1 \in H_{0}$. Now, assume that $x \rightarrow y, x \rightarrow(y \rightarrow z) \in H_{0}$. Then there exist $k, t \in(0,1]$ such that $\lambda(x \rightarrow y) \geq t$ and $\lambda(x \rightarrow(y \rightarrow z)) \geq k$, and so, $(x \rightarrow y)_{t} \in \lambda$ and $(x \rightarrow(y \rightarrow z))_{k} \in \lambda$. Thus, by Definition $4,(x \rightarrow z)_{\min \{t, k\}} \in \lambda$, so $\lambda(x \rightarrow z) \geq \min \{t, k\} \neq 0$. Hence, $x \rightarrow z \in H_{0}$. Therefore, $H_{0}$ is a positive implicative filter of $H$.

Proposition 3. Let $\lambda$ be an $(\in, \in)$-fuzzy positive implicative filter of $H$. Then $\lambda_{q}^{t}$ is a positive implicative filter of $H$ for any $t \in(0,1]$. 
Proof. Let $x \in \lambda_{q}^{t}$ for any $t \in(0,1]$ and $x \in H$. Then $x_{t} q \lambda$, and so $\lambda(x)+t>1$ Thus, $\lambda(x)>1-t$. By assumption, since $x_{1-t} \in \lambda$, we have $1_{1-t} \in \lambda$, so $\lambda(1)>1-t$. Hence, $\lambda(1)+t>1$, and so $1 \in \lambda_{q}^{t}$. Now, suppose $x \rightarrow y, x \rightarrow(y \rightarrow z) \in \lambda_{q}^{t}$ for any $x, y, z \in H$. Then

$$
\lambda(x \rightarrow y)+t>1, \lambda(x \rightarrow(y \rightarrow z))+t>1
$$

So

$$
\lambda(x \rightarrow y)>1-t, \lambda(x \rightarrow(y \rightarrow z))>1-t
$$

Since $\lambda$ is an $(\in, \in)$-fuzzy positive implicative filter of $H$, we have $\lambda(x \rightarrow z)>1-t$. Thus, $\lambda(x \rightarrow$ $z)+t>1$. Hence $x \rightarrow z \in \lambda_{q}^{t}$. Therefore $\lambda_{q}^{t}$ is a positive implicative filter of $H$.

Corollary 3. Let $\lambda$ be an $(\in, \in \vee q)$-fuzzy positive implicative filter of $H$. Then $\lambda_{\in \vee q}^{t}$ is a positive implicative filter of $H$, for any $t \in(0,1]$.

Proof. By Theorem 5 and Proposition 3, the proof is clear.

Theorem 6. If $x^{2}=x$, for any $x \in H$, then any $(\epsilon, \epsilon)$-fuzzy filter of $H$ is an $(\epsilon, \epsilon)$-fuzzy positive implicative filter.

Proof. Suppose that $H$ satisfies $x^{2}=x$ for all $x \in H$. Let $\lambda$ be an $(\in, \in)$-fuzzy filter of $H$ and let $x, y, z \in H$ and $t, k \in(0,1]$ be such that $(x \rightarrow(y \rightarrow z))_{t} \in \lambda$ and $(x \rightarrow y)_{k} \in \lambda$. By Proposition 1(vii), we get

$$
(x \rightarrow y) \leq(y \rightarrow(x \rightarrow z)) \rightarrow(x \rightarrow(x \rightarrow z)) .
$$

Corollary 1 implies that

$$
((y \rightarrow(x \rightarrow z)) \rightarrow(x \rightarrow(x \rightarrow z)))_{k} \in \lambda .
$$

Since $\lambda$ is an $(\in, \in)$-fuzzy filter of $H$, we have $(x \rightarrow(x \rightarrow z))_{\min \{t, k\}} \in \lambda$, and hence

$$
((x \odot x) \rightarrow z)_{\min \{t, k\}} \in \lambda .
$$

Since $x^{2}=x$ for any $x \in H$, we get $(x \rightarrow z)_{\min \{t, k\}} \in \lambda$. Therefore $\lambda$ is an $(\in, \in)$-fuzzy positive implicative filter of $H$.

Theorem 7. Let $\lambda$ be an $(\in, \in)$-fuzzy sub-hoop of $H$ such that $1_{t} \in \lambda$ for any $t \in(0,1]$. For any $t, k \in(0,1]$ and $x, y, z \in H$, the following statements are equivalent:

(i) $\lambda$ is an $(\in, \in)$-fuzzy positive implicative filter,

(ii) $\lambda$ is an $(\in, \in)$-fuzzy filter and $(x \rightarrow(x \rightarrow y))_{t} \in \lambda$ imply $(x \rightarrow y)_{t} \in \lambda$,

(iii) $\lambda$ is an $(\in, \in)$-fuzzy filter and $(z \rightarrow(y \rightarrow x))_{t} \in \lambda$ imply $((z \rightarrow y) \rightarrow(z \rightarrow x))_{t} \in \lambda$,

(iv) $1_{t} \in \lambda$ and if $(z \rightarrow(y \rightarrow(y \rightarrow x)))_{t} \in \lambda$ and $z_{k} \in \lambda$, then $(y \rightarrow x)_{\min \{t, k\}} \in \lambda$,

(v) $\lambda$ is an $(\in, \in)$-fuzzy filter and $\left(x \rightarrow x^{2}\right)_{t} \in \lambda$.

Proof. Let $x, y, z \in H$ and $t, k \in(0,1]$. Then

(i) $\Rightarrow($ ii) Let $\lambda$ be an $(\in, \in)$-fuzzy positive implicative filter. Then by Theorem $4, \lambda$ is an $(\in, \in)$-fuzzy filter. Now, let $(x \rightarrow(x \rightarrow y))_{t} \in \lambda$. Since $(x \rightarrow x)_{t}=1_{t} \in \lambda$, by (i), $(x \rightarrow y)_{t} \in \lambda$. 
(ii) $\Rightarrow\left(\right.$ i) Since $\lambda$ is an $(\in, \in)$-fuzzy filter, if $x_{t} \in \lambda$, then $1_{t} \in \lambda$. Now, let $(x \rightarrow(y \rightarrow z))_{t} \in \lambda$ and $(x \rightarrow y)_{k} \in \lambda$. By Proposition 1(vii) and Corollary 1 ,

$$
x \rightarrow y \leq(y \rightarrow(x \rightarrow z)) \rightarrow(x \rightarrow(x \rightarrow z))
$$

and so

$$
((x \rightarrow(y \rightarrow z)) \rightarrow(x \rightarrow(x \rightarrow z)))_{k} \in \lambda
$$

Since $\lambda$ is an $(\in, \in)$-fuzzy filter and $(x \rightarrow(y \rightarrow z))_{t} \in \lambda$, we have $(x \rightarrow(x \rightarrow z))_{\min \{t, k\}} \in \lambda$. Then by (ii), $(x \rightarrow z)_{\min \{t, k\}} \in \lambda$. Hence, $\lambda$ is an $(\in, \in)$-fuzzy positive implicative filter of $H$.

(ii) $\Rightarrow$ (iii) Let $(z \rightarrow(y \rightarrow x))_{t} \in \lambda$. Then $(y \rightarrow(z \rightarrow x))_{t} \in \lambda$. Thus, from $z \odot(z \rightarrow y) \leq y$ and Proposition 1(viii), we obtain

$$
y \rightarrow(z \rightarrow x) \leq(z \odot(z \rightarrow y)) \rightarrow(z \rightarrow x)
$$

Since $\lambda$ is an $(\in, \in)$-fuzzy filter, by Corollary 1 ,

$$
(z \rightarrow((z \rightarrow y) \rightarrow(z \rightarrow x)))_{t} \in \lambda
$$

and so

$$
(z \rightarrow(z \rightarrow((z \rightarrow y) \rightarrow x)))_{t} \in \lambda .
$$

By (ii), $(z \rightarrow((z \rightarrow y) \rightarrow x))_{t} \in \lambda$, and so $((z \rightarrow y) \rightarrow(z \rightarrow x))_{t} \in \lambda$.

(iii) $\Rightarrow\left(\right.$ iv) Since $\lambda$ is an $(\in, \in)$-fuzzy filter of $H$, if $x_{t} \in \lambda$, then $1_{t} \in \lambda$. Let $(z \rightarrow(y \rightarrow(y \rightarrow x)))_{t} \in \lambda$ and $z_{k} \in \lambda$. It follows that $(y \rightarrow(y \rightarrow x))_{\min \{t, k\}} \in \lambda$. Thus by (iii), $(y \rightarrow x)_{\min \{t, k\}} \in \lambda$.

(iv) $\Rightarrow$ (ii) Let $x_{t} \in \lambda$ and $(x \rightarrow y)_{k} \in \lambda$. Then

$$
(x \rightarrow y)_{k}=(x \rightarrow(1 \rightarrow(1 \rightarrow y)))_{k} \in \lambda
$$

Since $x_{t} \in \lambda$, by (iv), $(1 \rightarrow y)_{\min \{t, k\}}=y_{\min \{t, k\}} \in \lambda$. So, $\lambda$ is an $(\in, \in)$-fuzzy filter of $H$. Now, let $(x \rightarrow(x \rightarrow y))_{t} \in \lambda$. Since $\lambda$ is an $(\in, \in)$-fuzzy filter, $1_{t} \in \lambda$ and $(1 \rightarrow(x \rightarrow(x \rightarrow y)))_{t} \in \lambda$, by (iv) we have, $(x \rightarrow y)_{t} \in \lambda$.

(ii) $\Rightarrow(v)$ Since $((x \odot x) \rightarrow(x \odot x))_{t}=1_{t} \in \lambda$, we have $(x \rightarrow(x \rightarrow(x \odot x)))_{t}=1_{t} \in \lambda$. Then by (ii), $\left(x \rightarrow x^{2}\right)_{t} \in \lambda$.

$(v) \Rightarrow(i i)$ Let $(x \rightarrow(x \rightarrow y))_{t} \in \lambda$. Then $\left(x^{2} \rightarrow y\right)_{t} \in \lambda$ and by $(v),\left(x \rightarrow x^{2}\right)_{k} \in \lambda$. Since $\lambda$ is an $(\in, \in)$-fuzzy filter of $H$, by Proposition 1 (vii), we have $\left(x \rightarrow x^{2}\right) \leq\left(x^{2} \rightarrow y\right) \rightarrow(x \rightarrow y)$, and so $\left(\left(x^{2} \rightarrow y\right) \rightarrow(x \rightarrow y)\right)_{k} \in \lambda$. Therefore $(x \rightarrow y)_{\min \{t, k\}} \in \lambda$.

Theorem 8. Every $(\in, \in)$-fuzzy implicative filter of $H$ is an $(\in, \in)$-fuzzy positive implicative filter of $H$.

Proof. Suppose that $\lambda$ is an $(\in, \in)$-fuzzy implicative filter of $H$ and let $x_{t} \in \lambda$ for any $t \in(0,1]$ and $x \in H$. Then by Definition $3,1_{t} \in \lambda$. Now, let $x, y, z \in H$ and $t, k \in(0,1]$ such that $(z \rightarrow(y \rightarrow$ $(y \rightarrow x))))_{t} \in \lambda$ and $z_{k} \in \lambda$. Since $\lambda$ is an $(\in, \in)$-fuzzy implicative filter of $H$, by Theorem $2, \lambda$ is an $(\in, \in)$-fuzzy filter of $H$, so $(y \rightarrow(y \rightarrow x))_{\min \{t, k\}} \in \lambda$. Also, by Proposition 1 (vii), we have,

$$
y \rightarrow(y \rightarrow x) \leq((y \rightarrow x) \rightarrow x) \rightarrow(y \rightarrow x) .
$$

Then by Corollary 1,

$$
((y \rightarrow(y \rightarrow x)) \rightarrow(((y \rightarrow x) \rightarrow x) \rightarrow(y \rightarrow x)))_{\min \{t, k\}}=1_{\min \{t, k\}} \in \lambda
$$


Since $\lambda$ is an $(\in, \in)$-fuzzy implicative filter of $H,(y \rightarrow x)_{\min \{t, k\}} \in \lambda$. Hence, by Theorem $7, \lambda$ is an $(\in, \in)$-fuzzy positive implicative filter of $H$.

In the following example we show that the converse of Theorem 8, may not be true, in general.

Example 3. Let $H$ be a hoop as Example 1. Define $\lambda(1)=0.6, \lambda(a)=0.4, \lambda(b)=0.3$ and $\lambda(0)=0.5$. By routine calculations, we can verify that $\lambda$ is an $(\in, \in)$-fuzzy positive implicative filter of $H$. But it is not an $(\in, \in)$-fuzzy implicative filter of $H$ since $((b \rightarrow 0) \rightarrow b)_{0.8}=1_{0.8} \in \lambda$, but $b_{0.8} \notin \lambda$.

Theorem 9. Let $\lambda$ be an $(\in, \in)$-fuzzy positive implicative filter of $H$. Then $\lambda$ is an $(\in, \in)$-fuzzy implicative filter if and only if, for any $t \in(0,1]$ and $x, y \in H$, if $((x \rightarrow y) \rightarrow y)_{t} \in \lambda$, then $((y \rightarrow x) \rightarrow x)_{t} \in \lambda$.

Proof. $(\Rightarrow)$ Let $\lambda$ be an $(\in, \in)$-fuzzy implicative filter of $H$ and $((x \rightarrow y) \rightarrow y)_{t} \in \lambda$ for all $x, y \in H$ and $t \in(0,1]$. By Proposition 1(x), $y^{\prime} \leq x \rightarrow y$. Then by Proposition 1(viii), $(x \rightarrow y) \rightarrow y \leq y^{\prime} \rightarrow y$. Also, by Theorem $4, \lambda$ is an $(\in, \in)$-fuzzy filter of $H$, then by Corollary $1,\left(y^{\prime} \rightarrow y\right)_{t} \in \lambda$. Moreover, by Proposition $1(\mathrm{v}),\left(1 \rightarrow\left(y^{\prime} \rightarrow y\right)\right)_{t} \in \lambda$. Since $\lambda$ is an $(\in, \in)$-fuzzy implicative filter, then $y_{t} \in \lambda$. On the other side, by Proposition 1(iv), $y \leq(y \rightarrow x) \rightarrow x$. Since $\lambda$ is an $(\in, \in)$-fuzzy filter, then by Corollary 1 , $((y \rightarrow x) \rightarrow x)_{t} \in \lambda$.

$(\Leftarrow)$ Let $((x \rightarrow y) \rightarrow x)_{t} \in \lambda$ for all $x, y \in H$ and $t \in(0,1]$. Using Proposition 1 (vi) and (viii), we get $x \leq(x \rightarrow y) \rightarrow y$, and thus

$$
(x \rightarrow y) \rightarrow x \leq(x \rightarrow y) \rightarrow((x \rightarrow y) \rightarrow y) .
$$

By Theorem $4, \lambda$ is an $(\in, \in)$-fuzzy filter, then by Corollary $1,((x \rightarrow y) \rightarrow((x \rightarrow y) \rightarrow y))_{t} \in \lambda$. Since $\lambda$ is an $(\in, \in)$-fuzzy positive implicative filter and $((x \rightarrow y) \rightarrow(x \rightarrow y))_{t}=1_{t} \in \lambda$, then by Theorem 7, $((x \rightarrow y) \rightarrow y)_{t} \in \lambda$, and so by assumption, $((y \rightarrow x) \rightarrow x)_{t} \in \lambda$. Moreover, by Proposition 1(iv), $y \leq x \rightarrow y$. Also, we have $(x \rightarrow y) \rightarrow x \leq y \rightarrow x$ by Proposition 1(viii). Since $((x \rightarrow y) \rightarrow x)_{t} \in \lambda$ and $\lambda$ is an $(\in, \in)$-fuzzy filter, then by Corollary $1,(y \rightarrow x)_{t} \in \lambda$. Hence, $((y \rightarrow x) \rightarrow x)_{t} \in \lambda$ and $(y \rightarrow x)_{t} \in \lambda$. Since $\lambda$ is an $(\in, \in)$-fuzzy filter, then $x_{t} \in \lambda$. Therefore, by ([25] Theorem 3.7), $\lambda$ is an $(\in, \in)$-fuzzy implicative filter.

Corollary 4. $\lambda$ is an $(\in, \in)$-fuzzy implicative filter if and only if $\lambda$ is an $(\in, \in)$-fuzzy positive implicative filter such that if $((x \rightarrow y) \rightarrow y)_{t} \in \lambda$, then $((y \rightarrow x) \rightarrow x)_{t} \in \lambda$, for any $x, y \in H$ and $t \in(0,1]$.

Theorem 10. Let $\lambda$ be an $(\in, \in)$-fuzzy filter of $H$. Then $\lambda$ is an $(\in, \in)$-fuzzy positive implicative filter if and only if $\frac{H}{\equiv_{\lambda}}$ is a Brouwerian semilattice.

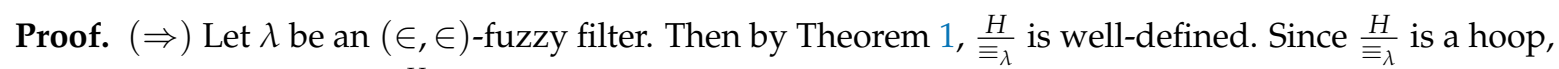
then by Proposition 1(i), $\stackrel{H}{\equiv}$ is a $\wedge$-semilattice. Now, it is enough to prove that

$$
[x]_{\lambda} \wedge[y]_{\lambda} \leq[z]_{\lambda} \text { if and only if }[x]_{\lambda} \leq[y]_{\lambda} \rightsquigarrow[z]_{\lambda} \text {, for all } x, y, z \in H
$$

Let $[x]_{\lambda} \wedge[y]_{\lambda} \leq[z]_{\lambda}$. Then by Proposition 1(iii), $[x]_{\lambda} \otimes[y]_{\lambda} \leq[x]_{\lambda} \wedge[y]_{\lambda} \leq[z]_{\lambda}$. Thus, $[x]_{\lambda} \otimes[y]_{\lambda} \leq$ $[z]_{\lambda}$. Since $\frac{H}{\equiv_{\lambda}}$ is a hoop, by Proposition 1 (ii), $[x]_{\lambda} \leq[y]_{\lambda} \rightsquigarrow[z]_{\lambda}$.

Conversely, suppose $[x]_{\lambda} \leq[y]_{\lambda} \rightsquigarrow[z]_{\lambda}$. Then by Theorem $1,(x \rightarrow(y \rightarrow z))_{t} \in \lambda$, for $t \in(0,1]$. Since $\lambda$ is an $(\in, \in)$-fuzzy positive implicative filter, by Theorem 7(iii), $((x \rightarrow y) \rightarrow(x \rightarrow z))_{t} \in \lambda$. Thus, $[x \rightarrow y]_{\lambda} \leq[x \rightarrow z]_{\lambda}$. Hence, $[x]_{\lambda} \rightsquigarrow[y]_{\lambda} \leq[x]_{\lambda} \rightsquigarrow[z]_{\lambda}$. Since $\frac{H}{\equiv_{\lambda}}$ is a hoop, by Proposition 1(ii) and (i),

$$
[x]_{\lambda} \wedge[y]_{\lambda}=[x]_{\lambda} \otimes\left([x]_{\lambda} \rightsquigarrow[y]_{\lambda}\right) \leq[z]_{\lambda}
$$


Therefore, $\frac{H}{\equiv}$ is a Brouwerian semilattice.

$(\Leftarrow)$ Since $\lambda$ is an $(\in, \in)$-fuzzy filter of $H$, if $x_{t} \in \lambda$, for $x \in H$ and $t \in(0,1]$, then $1_{t} \in \lambda$. By assumption, $\stackrel{H}{\equiv_{\lambda}}$ is a Brouwerian semilattice, define $[x]_{\lambda} \otimes[y]_{\lambda}=[x]_{\lambda} \wedge[y]_{\lambda}$, for all $x, y \in H$. Then

$$
[x]_{\lambda} \leq[x]_{\lambda} \wedge[x]_{\lambda}=[x]_{\lambda} \otimes[x]_{\lambda}=[x \odot x]_{\lambda}
$$

So, $[x]_{\lambda} \leq\left[x^{2}\right]_{\lambda}$. By Theorem $1,\left(x \rightarrow x^{2}\right)_{t} \in \lambda$. Hence, by Theorem $7(\mathrm{v}), \lambda$ is an $(\in, \in)$-fuzzy positive implicative filter.

Note. According to Aaly Kologani et al. [19], every $(\in, \in)$-fuzzy sub-hoop is an $(\in, \in \vee q)$-fuzzy sub-hoop of $H$. Easily we can consequence that every $(\epsilon, \in)$-fuzzy positive implicative filter of $H$ is an $(\in, \in \vee q)$-fuzzy positive implicative filter of $H$. But by Example ([25] Example 3.9), there exists $(\in, \in \vee q)$-fuzzy positive implicative filter of $H$ that is not an $(\in, \in \vee q)$-fuzzy filter. So some of above theorem that proved in this section, hold for $(\in, \in \vee q)$-fuzzy positive implicative filter of $H$. But some of them hold with conditions, because of that we prove them again.

Theorem 11. A fuzzy set $\lambda$ in $H$ is an $(\in, \in \vee q)$-fuzzy positive implicative filter of $H$ if and only if, for any $t \in(0,0.5]$ it satisfies:

$$
\begin{aligned}
& (\forall x \in H)(\lambda(1) \geq \min \{\lambda(x), 0.5\}) \\
& (\forall x, y \in H)(\lambda(x \rightarrow z) \geq \min \{\lambda(x \rightarrow y), \lambda(x \rightarrow(y \rightarrow z)), 0.5\}) .
\end{aligned}
$$

Proof. Let $\lambda$ be an $(\in, \in \vee q)$-fuzzy positive implicative filter of $H$ and $x \in H$ such that $\lambda(x)=t$, so $x_{t} \in \lambda$. Since $\lambda$ is an $(\in, \in \vee q)$-fuzzy positive implicative filter of $H$, we have $1_{t} \in \vee q \lambda$. If $1_{t} \in \lambda$, then $\lambda(1) \geq t=\lambda(x)$ and if $1_{t} q \lambda$, then $\lambda(1)+t>1$, and so $\lambda(1)>1-t$. Since $t \in(0,0.5]$, it is clear that $\lambda(1)>1-t>t$. Hence, for any $x \in H, \lambda(1) \geq \lambda(x)$. Therefore, $\lambda(1) \geq \min \{\lambda(x), 0.5\}$. Now, let $x, y, z \in H$ and $t, k \in(0,0.5]$ such that $\lambda(x \rightarrow y) \geq t$ and $\lambda(x \rightarrow(y \rightarrow z)) \geq k$, so $(x \rightarrow y)_{t} \in \lambda$ and $(x \rightarrow(y \rightarrow z))_{k} \in \lambda$. Since $\lambda$ is an $(\in, \in \vee q)$-fuzzy positive implicative filter of $H$, $(x \rightarrow z)_{\min \{t, k\}} \in \vee q \lambda$. If $(x \rightarrow z)_{\min \{t, k\}} \in \lambda$, then

$$
\lambda(x \rightarrow z) \geq \min \{\lambda(x \rightarrow y), \lambda(x \rightarrow(y \rightarrow z))\}
$$

If $(x \rightarrow z)_{\min \{t, k\}} q \lambda$, then $\lambda(x \rightarrow z)+\min \{t, k\}>1$. Since $t \in(0,0.5]$, it is clear that $\lambda(x \rightarrow z)>$ $1-\min \{t, k\}$, and so $\lambda(x \rightarrow z) \geq \min \{t, k\}$. Hence, in both cases

$$
\min \{\lambda(x \rightarrow(y \rightarrow z)), \lambda(x \rightarrow y), 0.5\} \leq \lambda(x \rightarrow z) .
$$

Conversely, the proof is similar to the proof of Theorem 3.

Theorem 12. Every $(\in, \in \vee q)$-fuzzy positive implicative filter of $H$ is an $(\in, \in \vee q)$-fuzzy filter of $H$.

Proof. The proof is similar to the proof of Theorem 4.

Corollary 5. Every $(\in, \in)$-fuzzy positive implicative filter of $H$ such as $\lambda$ satisfies the following assertion:

$$
(\forall x, y \in H)(\text { if } x \leq y \text {, then } \lambda(x) \leq \lambda(y))
$$


Proof. According to Theorem 3, since $\lambda$ is an $(\epsilon, \in \vee q)$-fuzzy positive implicative filter of $H$, we have $\lambda(x) \leq \lambda(1)$, for $x \in H$ and $t \in(0,0.5]$. Since $x \leq y$, we have $x \rightarrow y=1$. Then by Theorem 11, we get that

$$
\begin{aligned}
\lambda(y) & =\lambda(1 \rightarrow y) \geq \min \{\lambda(1 \rightarrow x), \lambda(1 \rightarrow(x \rightarrow y))\} \\
& =\min \{\lambda(x), \lambda(x \rightarrow y)\}=\min \{\lambda(x), \lambda(1)\} \\
& =\lambda(x)
\end{aligned}
$$

Theorem 13. If $x^{2}=x$, for any $x \in H$, then any $(\in, \in \vee q)$-fuzzy filter of $H$ is an $(\in, \in \vee q)$-fuzzy positive implicative filter of $H$, for any $t \in(0,0.5]$.

Proof. Suppose, for any $x \in H, x^{2}=x$. Let $\lambda$ be an $(\in, \in \vee q)$-fuzzy filter of $H,(x \rightarrow(y \rightarrow z))_{t} \in \lambda$ and $(x \rightarrow y)_{k} \in \lambda$, for any $x, y, z \in H$ and $t, k \in(0,0.5]$. By Proposition 1 (vii),

$$
(x \rightarrow y) \leq(y \rightarrow(x \rightarrow z)) \rightarrow(x \rightarrow(x \rightarrow z)) .
$$

Since $\lambda$ is an $(\in, \in \vee q)$-fuzzy filter of $H$, by Corollary 1 ,

$$
\lambda(x \rightarrow(x \rightarrow z))) \geq \min \{\lambda(x \rightarrow y), \lambda(y \rightarrow(x \rightarrow z)), 0.5\}
$$

and so $((x \odot x) \rightarrow z)_{\min \{t, k\}} \in \vee q \lambda$. Since $x^{2}=x$, for any $x \in H,(x \rightarrow z)_{\min \{t, k\}} \in \vee q \lambda$. Therefore, $\lambda$ is an $(\in, \in \vee q)$-fuzzy positive implicative filter of $H$.

Theorem 14. Let $\lambda$ be an $(\in, \in \vee q)$-fuzzy sub-hoop of $H$ such that $1_{t} \in \lambda$, for any $t \in(0,0.5]$. Then, for any $x, y, z \in H$ and $t, k \in(0,0.5]$, the following statements are equivalent:

(i) $\lambda$ is an $(\in, \in \vee q)$-fuzzy positive implicative filter of $H$,

(ii) $\lambda$ is an $(\in, \in \vee q)$-fuzzy filter and $\min \{\lambda(x \rightarrow(x \rightarrow y)), 0.5\} \leq \lambda(x \rightarrow y)$,

(iii) $\lambda$ is an $(\in, \in \vee q)$-fuzzy filter and $\min \{\lambda(z \rightarrow(y \rightarrow x)), 0.5\} \leq \lambda((z \rightarrow y) \rightarrow(z \rightarrow x))$,

(iv) $1_{t} \in \lambda$ and $\min \{\lambda(z \rightarrow(y \rightarrow(y \rightarrow x))), \lambda(z), 0.5\} \leq \lambda(y \rightarrow x)$,

(v) $\lambda$ is an $(\in, \in \vee q)$-fuzzy filter and $\min \{\lambda(1), 0.5\} \leq \lambda\left(x \rightarrow x^{2}\right)$.

Proof. Let $x, y, z \in H$. Then

(i) $\Rightarrow$ (ii) Let $\lambda$ be an $(\in, \in \vee q)$-fuzzy positive implicative filter of $H$. Then by Theorem $12, \lambda$ is an $(\in, \in \vee q)$-fuzzy filter of $H$. Now, let $(x \rightarrow(x \rightarrow y))_{t} \in \lambda$. Then $(x \rightarrow x)_{t}=1_{t} \in \lambda$, so by (i), $\min \{\lambda(x \rightarrow(x \rightarrow y)), 0.5\} \leq \lambda(x \rightarrow y)$.

(ii) $\Rightarrow($ i $)$ Since $\lambda$ is an $(\in, \in \vee q)$-fuzzy filter of $H$, if $x_{t} \in \lambda$, then $1_{t} \in \vee q \lambda$. Now, let $(x \rightarrow(y \rightarrow z))_{t} \in$ $\lambda$ and $(x \rightarrow y)_{k} \in \lambda$. By Proposition 1(vii) and Corollary 1 ,

$$
x \rightarrow y \leq(y \rightarrow(x \rightarrow z)) \rightarrow(x \rightarrow(x \rightarrow z))
$$

and so

$$
\min \{\lambda(x \rightarrow y), 0.5\} \leq \lambda((x \rightarrow(y \rightarrow z)) \rightarrow(x \rightarrow(x \rightarrow z))) .
$$

Since $\lambda$ is an $(\in, \in \vee q)$-fuzzy filter of $H$ and $(x \rightarrow(y \rightarrow z))_{t} \in \lambda$, we have

$$
\min \{\lambda((x \rightarrow(y \rightarrow z)) \rightarrow(x \rightarrow(x \rightarrow z))), \lambda(x \rightarrow(y \rightarrow z)), 0.5\} \leq \lambda(x \rightarrow(x \rightarrow z)) .
$$


Then by (ii), $\min \{\lambda(x \rightarrow(x \rightarrow z)), 0.5\} \leq \lambda(x \rightarrow z)$. Hence,

$$
\begin{aligned}
& \min \{\lambda(x \rightarrow y), \lambda(x \rightarrow(y \rightarrow z)), 0.5\} \\
\leq & \min \{\lambda((x \rightarrow(y \rightarrow z)) \rightarrow(x \rightarrow(x \rightarrow z))), \lambda(x \rightarrow(y \rightarrow z)), 0.5\} \\
\leq & \min \{\lambda(x \rightarrow(x \rightarrow z)), 0.5\} \\
\leq & \lambda(x \rightarrow z)
\end{aligned}
$$

Therefore, $\lambda$ is an $(\in, \in \vee q)$-fuzzy positive implicative filter of $H$.

The proof of other cases is similar to Theorem 7 and $(i) \Leftrightarrow(i i)$.

Theorem 15. If $\lambda$ is a non-zero $(\in, \in \vee q)$-fuzzy positive implicative filter of $H$, then the set

$$
H_{0}:=\{x \in H \mid \lambda(x) \neq 0\}
$$

is a positive implicative filter of $\mathrm{H}$.

Proof. The proof is similar to Theorem 5 .

Proposition 4. Let $\lambda$ be an $(\in, \in \vee q)$-fuzzy positive implicative filter of $H$. Then $\lambda_{q}^{t}$ is a positive implicative filter of $H$ for any $t \in(0.5,1]$.

Proof. Let $x \in \lambda_{q}^{t}$ for any $t \in(0.5,1]$ and $x \in H$. Then $x_{t} q \lambda$, and so $\lambda(x)+t>1$. Thus, $\lambda(x)>1-t$. By assumption, since $x_{1-t} \in \lambda$, we have $1_{1-t} \in \vee q \lambda$. If $\lambda(1)>1-t$, then $\lambda(1)+t>1$, and so $1 \in \lambda_{q}^{t}$. If $\lambda(1)+1-t>1$, then $\lambda(1)>t$. Since $t \in(0.5,1]$, we have $\lambda(1)+t>2 t>1$. Hence, $\lambda(1)+t>1$, and so $1 \in \lambda_{q}^{t}$. Now, suppose $x \rightarrow y, x \rightarrow(y \rightarrow z) \in \lambda_{q}^{t}$, for any $x, y, z \in H$ and $t \in(0.5,1]$. Then $\lambda(x \rightarrow y)+t>1$ and $\lambda(x \rightarrow(y \rightarrow z))+t>1$, so $\lambda(x \rightarrow y)>1-t$ and $\lambda(x \rightarrow(y \rightarrow z))>1-t$. Since $\lambda$ is an $(\in, \in \vee q)$-fuzzy positive implicative filter of $H$, we have $\lambda(x \rightarrow z)>1-t$ or $\lambda(x \rightarrow z)+1-t>1$. If $\lambda(x \rightarrow z)>1-t$, then $\lambda(x \rightarrow z)+t>1$ and if $\lambda(x \rightarrow z)>t$, since $t \in(0.5,1]$, then $\lambda(x \rightarrow z)+t>2 t>1$. Thus, in both cases, $\lambda(x \rightarrow z)+t>1$. Hence, $x \rightarrow z \in \lambda_{q}^{t}$. Therefore, $\lambda_{q}^{t}$ is a positive implicative filter of $H$.

Corollary 6. Let $\lambda$ be an $(\in, \in \vee q)$-fuzzy positive implicative filter of $H$. Then $\lambda_{\in \vee}^{t}$ is a positive implicative filter of $H$, for any $t \in(0,1]$.

Proof. By Theorem 15 and Proposition 4, the proof is clear.

Theorem 16. Every $(\in, \in \vee q)$-fuzzy implicative filter of $H$ is an $(\in, \in \vee q)$-fuzzy positive implicative filter of $H$.

Proof. Let $\lambda$ be an $(\in, \in \vee q)$-fuzzy implicative filter of $H$. Then by Proposition 2, for any $x, y \in H$, $\min \{\lambda(x \rightarrow(x \rightarrow y)), 0.5\} \leq \lambda(x \rightarrow y)$. Thus, by Theorem $14, \lambda$ is an $(\in, \in \vee q)$-fuzzy positive implicative filter of $H$.

Theorem 17. Let $\lambda$ be an $(\in, \in \vee q)$-fuzzy positive implicative filter of $H$. Then $\lambda$ is an $(\in, \in \vee q)$-fuzzy implicative filter if and only if, for any $x, y \in H$, if $\min \{\lambda(1), 0.5\} \leq \lambda((x \rightarrow y) \rightarrow y)$, then $\min \{\lambda(1), 0.5\} \leq \lambda((y \rightarrow x) \rightarrow x)$. 
Proof. $(\Rightarrow)$ Assume that $\lambda$ is an $(\in, \in \vee q)$-fuzzy implicative filter of $H$ and $\min \{\lambda(1), 0.5\} \leq \lambda((x \rightarrow$ $y) \rightarrow y$ ) for all $x, y \in H$. By Proposition 1(x), we have $y^{\prime} \leq x \rightarrow y$. Then by Proposition 1(viii), we obtain $(x \rightarrow y) \rightarrow y \leq y^{\prime} \rightarrow y$. By Theorem $12, \lambda$ is an $(\in, \in \vee q)$-fuzzy filter of $H$, then by Corollary 5 ,

$$
\min \{\lambda((x \rightarrow y) \rightarrow y), 0.5\} \leq \lambda\left(y^{\prime} \rightarrow y\right) .
$$

By Proposition 1(v),

$$
\min \{\lambda((x \rightarrow y) \rightarrow y), 0.5\} \leq \lambda\left(1 \rightarrow\left(y^{\prime} \rightarrow y\right)\right) .
$$

Since $\lambda$ is an $(\in, \in \vee q)$-fuzzy implicative filter of $H, \min \left\{\lambda\left(1 \rightarrow\left(y^{\prime} \rightarrow y\right)\right), 0.5\right\} \leq \lambda(y)$. Moreover, by Proposition 1(iv), $y \leq(y \rightarrow x) \rightarrow x$. Since $\lambda$ is an $(\in, \in \vee q)$-fuzzy filter of $H$, by Corollary 5 , $\min \{\lambda(y), 0.5\} \leq \lambda((y \rightarrow x) \rightarrow x)$. Hence

$$
\begin{aligned}
\min \{\lambda(1), 0.5\} & \leq \min \{\lambda((x \rightarrow y) \rightarrow y), 0.5\} \\
& \leq \min \left\{\lambda\left(1 \rightarrow\left(y^{\prime} \rightarrow y\right)\right), 0.5\right\} \\
& \leq \min \{\lambda(y), 0.5\} \\
& \leq \lambda((y \rightarrow x) \rightarrow x)
\end{aligned}
$$

Therefore, $\min \{\lambda(1), 0.5\} \leq \lambda((y \rightarrow x) \rightarrow x)$.

$(\Leftarrow)$ Let $\min \{\lambda(1), 0.5\} \leq \lambda((x \rightarrow y) \rightarrow x)$, for all $x, y \in H$. By Proposition 1 (vi) and (viii), $x \leq(x \rightarrow$ $y) \rightarrow y$, and so

$$
(x \rightarrow y) \rightarrow x \leq(x \rightarrow y) \rightarrow((x \rightarrow y) \rightarrow y)
$$

By Theorem $12, \lambda$ is an $(\in, \in \vee q)$-fuzzy filter of $H$, then by Corollary 5 ,

$$
\min \{\lambda((x \rightarrow y) \rightarrow x), 0.5\} \leq \lambda((x \rightarrow y) \rightarrow((x \rightarrow y) \rightarrow y))
$$

Since $\lambda$ is an $(\in, \in \vee q)$-fuzzy positive implicative filter and $((x \rightarrow y) \rightarrow(x \rightarrow y))_{t}=1_{t} \in \lambda$, by Theorem 14,

$$
\begin{aligned}
\min \{\lambda(1), 0.5\} & \leq \min \{\lambda((x \rightarrow y) \rightarrow x), 0.5\} \\
& \leq\{\lambda((x \rightarrow y) \rightarrow((x \rightarrow y) \rightarrow y), 0.5\} \\
& \leq \lambda((x \rightarrow y) \rightarrow y)
\end{aligned}
$$

and so by assumption, $\min \{\lambda(1), 0.5\} \leq \lambda((y \rightarrow x) \rightarrow x)$. Moreover, by Proposition 1(iv), $y \leq x \rightarrow y$. Also, by Proposition 1(viii), $(x \rightarrow y) \rightarrow x \leq y \rightarrow x$. Since $\min \{\lambda(1), 0.5\} \leq \lambda((x \rightarrow y) \rightarrow x)$ and $\lambda$ is an $(\in, \in \vee q)$-fuzzy filter of $H$, by Corollary $1, \min \{\lambda(1), 0.5\} \leq \lambda(y \rightarrow x)$. Hence, $\min \{\lambda(1), 0.5\} \leq$ $\lambda((y \rightarrow x) \rightarrow x)$ and $\min \{\lambda(1), 0.5\} \leq \lambda(y \rightarrow x)$. Since $\lambda$ is an $(\in, \in \vee q)$-fuzzy filter of $H$, we obtain that $\min \{\lambda(1), 0.5\} \leq \lambda(x)$. Therefore, by [25, Theorem 4.7], $\lambda$ is an $(\in, \in \vee q)$-fuzzy implicative filter of $H$.

Corollary 7. $\lambda$ is an $(\in, \in \vee q)$-fuzzy implicative filter of $H$ if and only if $\lambda$ is an $(\in, \in \vee q)$-fuzzy positive implicative filter of $H$ such that if $\min \{\lambda(1), 0.5\} \leq \lambda((x \rightarrow y) \rightarrow y)$, then $\min \{\lambda(1), 0.5\} \leq \lambda((y \rightarrow$ $x) \rightarrow x)$, for any $x, y \in H$.

Theorem 18. Let $\lambda$ be an $(\in, \in \vee q)$-fuzzy filter of $H$. Then $\lambda$ is an $(\in, \in \vee q)$-fuzzy positive implicative filter of $H$ if and only if $\frac{H}{\equiv_{\lambda}}$ is a Brouwerian semilattice.

Proof. It is similar to the proof of Theorem 10. 


\section{Conclusions}

We have defined the notions of $(\in, \in \vee q)$-fuzzy positive implicative filters and $(\in, \in)$-fuzzy positive implicative filters of hoops, and have investigated some equivalent definitions and properties of them. We have shown that every $(\epsilon, \in \vee q)$-fuzzy implicative filter of $H$ is an $(\in, \in \vee q)$-fuzzy positive implicative filter of $H$. Using the congruence relation, we have shown that $\lambda$ is an $(\in, \in$ $\vee q)$-fuzzy positive implicative filter of $H$ if and only if $\frac{H}{\bar{\equiv}_{\lambda}}$ is a Brouwerian semilattice.

In the future works, we will introduce $(\alpha, \beta)$-fuzzy fantastic filters for $(\alpha, \beta) \in\{(\in, \in),(\in, \in \vee q)\}$ of hoops and investigate some properties of them. Also, we will study the relation between $(\alpha, \beta)$-fuzzy positive implicative filter and $(\alpha, \beta)$-fuzzy fantastic. Moreover, we will try to make a quotient structure by using these notions.

Author Contributions: R.A.B. and M.A.K. wrote the draft version of the paper, M.S.K. found and checked examples and Y.B.J. wrote the revised version and completed submission of the paper.

Funding: This research received no external funding.

Acknowledgments: The authors wish to thank the anonymous reviewers for their valuable suggestions.

Conflicts of Interest: The authors declare no conflict of interest.

\section{References}

1. Bosbach, B. Komplementäre Halbgruppen, Axiomatik und Arithmetik. Fundam. Math. 1969, 64, 257-287. [CrossRef]

2. Bosbach, B. Komplementäre Halbgruppen, Kongruenzen and Quotienten. Fundam. Math. 1970, 69, 1-14. [CrossRef]

3. Blok, W.J.; Ferreirim, I.M.A. On the structure of hoops. Algebra Univers. 2000, 43, 233-257. [CrossRef]

4. Blok, W.J.; Ferreirim, I.M.A. Hoops and their implicational reducts. Log. Comput. Sci. 1993, 28, 219-230.

5. Borzooei, R.A.; Aaly Kologani, M. Filter theory of hoop-algebras. J. Adv. Res. Pure Math. 2014, 6, 72-86. [CrossRef]

6. Alavia, S.Z.; Borzooei, R.A.; Aaly Kologani, M. Fuzzy filters in pseudo hoops. J. Intell. Fuzzy Syst. 2017, 32, 1997-2007. [CrossRef]

7. Ghorbani, S. Localization of hoop-algebras. J. Adv. Res. Pure Math. 2013, 5, 1-13. [CrossRef]

8. Luo, C.F.; Xin, X.L.; He, P. $n$-fold (positive) implicative filters of hoops. Ital. J. Pure Appl. Math. 2017, 38, 631-642.

9. Khorami, R.T.; Saeid, A.B. Some unitary operators on hoop-algebras. Fuzzy Inf. Eng. 2017, 9, 205-223. [CrossRef]

10. Borzooei, R.A.; Varasteh, H.R.; Borna, K. Fundamental hoop-algebras. Ratio Math. 2015, 29, 25-40.

11. Zander, M.A. Decomposability of the finitely generated free hoop residuation algebra. Studia Log. 2008, 88, 233-246. [CrossRef]

12. Pu, P.M.; Liu, Y.M. Fuzzy topology I, Neighborhood structure of a fuzzy point and Moore-Smith convergence. J. Math. Anal. Appl. 1980, 76, 571-599.

13. Jun, Y.B. On $(\alpha, \beta)$-fuzzy subalgebras of BCK/BCI-algebras. Bull. Korean Math. Soc. 2005, 42, 703-711. [CrossRef]

14. Broumand Saeid, A. Redefined fuzzy subalgebra (with thresholds) of BCK/BCI-algebras. Iran. J. Math. Sci. Inf. 2009, 4, 9-24.

15. Chiranjibe, J.; Madhumangal, P.; Borumand Saied, A. $(\in, \in \vee q)$-bipolar fuzzy BCK/BCI-algebras. Mo. J. Math. Sci. 2017, 29, 139-160.

16. Chiranjibe, J.; Madhumangal, P. On $\left(\in_{\alpha}, \in_{\alpha} \vee q_{\alpha}\right)$-fuzzy soft BCI-algebras. Mo J. Math. Sci. 2017, 29, 197-215.

17. Bakhshi, M.; Zare, M. $(\alpha, \beta)$-fuzzy ideals in pseudo MV-algebras. In Proceedings of the 12th Iranian Conference on Fuzzy Systems University of Mazandaran, Babolsar, Iran, 23-25 October 2012.

18. Al-Masarwah, A.; Ahmad, A.G. m-polar $(\alpha, \beta)$-fuzzy ideals in BCK/BCI-algebras. Symmetry 2019, 11, 44. [CrossRef]

19. Aaly Kologani, M.; Mohseni Takallo, M.; Kim, H.S. Fuzzy filters of hoops based on fuzzy points. Mathematics 2019, 7, 430. [CrossRef] 
20. Hasankhani, A.; Saadat, H. Some quotients on a BCK-algebra Generated by a fuzzy set. Iran. J. Fuzzy Syst. 2004, 1, 33-43.

21. Hoo, C.S. Fuzzy ideals of BCI and MV-algebras. Fuzzy Sets Syst. 1994, 62, 111-114. [CrossRef]

22. Kim, H.S.; Neggers, J.; Ahn, S.S. Almost $\phi$-fuzzy semi-ideals in groupoids. J. Intell. Fuzzy Syst. 2019, 36, 5361-5368. [CrossRef]

23. Liu, L.; Li, K. Fuzzy filters of BL-algebras. Inf. Sci. 2005, 173, 141-154. [CrossRef]

24. Georgescu, G.; Leustean, L.; Preoteasa, V. Pseudo-hoops. J. Mult.-Valued Log. Soft Comput. 2005, 11, $153-184$.

25. Aaly Kologani, M.; Xin, X.L.; Mohseni Takallo, M.; Jun, Y.B.; Borzooei, R.A. Fuzzy implicative filters of hoops based on fuzzy points. J. Intell. Fuzzy Syst. 2019, 7, 430.

(C) 2019 by the authors. Licensee MDPI, Basel, Switzerland. This article is an open access article distributed under the terms and conditions of the Creative Commons Attribution (CC BY) license (http://creativecommons.org/licenses/by/4.0/). 